\title{
Magnetic Tagging of Therapeutic Cells for MRI
}

Transplantation of stem cells or immune cells has shown promise for the treatment of several diseases. Monitoring magnetically labeled cells with MRI has furthered our understanding of cellular migration and the pathophysiology of diseases in experimental models. These studies should pave the way for guiding clinical trials using cell-based therapies. This review briefly describes the various methods used to label and track cells with MRI and the potential for such methods to translate to human applications.

Key Words: molecular imaging; MRl; magnetic cellular tagging; immune cells; stem cells

J Nucl Med 2009; 50:171-174

DOI: 10.2967/jnumed.108.053546

Cellular therapies have garnered considerable interest as potential treatments of diseases and for regenerative medicine. However, ensuring that cells reach their intended targets will be necessary to maximize the efficacy of these treatments. This has motivated the development of noninvasive imaging techniques to monitor the temporal and spatial migration of cells to target tissues. MRI is a well-suited tool for in vivo cell tracking because of its high spatial resolution. Numerous methods have been developed and refined in experimental models to track and monitor cells in vivo. Nearly all approaches require that cells be manipulated in vitro, either through the loading of cells with nanoparticles or through genetic modifications $(1-3)$. Recently, the use of superparamagnetic iron oxide nanoparticles (SPIONs) to visualize cell migration has been applied clinically, demonstrating the potential capabilities of monitoring cellular therapies with MRI. This concise review describes the methods used to label cells for tracking with in vivo MRI, with an emphasis on potential clinical translation. The readers are encouraged to examine the excellent published reviews ( $1-3)$ for comprehensive coverage of the many aspects of labeling cells for detection with MRI.

\section{METHODS OF CELL LABELING}

The labeling of mammalian cells for detection with MRI is achieved by loading the stem cells or immune cells in vitro with either paramagnetic contrast agents (i.e., gadolinium chelates), experimental or clinically approved SPIONs used off-label, or perfluorocarbon nanoparticles.

Received Aug. 29, 2008; revision accepted Nov. 25, 2008.

For correspondence or reprints contact: Matthew D. Budde, Radiology and Imaging Sciences, National Institutes of Health Clinical Center, Building 10,

Room B1N256, 10 Center Dr., MSC 1074, Bethesda, MD 20892.

E-mail: buddem@cc.nih.gov

COPYRIGHT @ 2009 by the Society of Nuclear Medicine, Inc.

\section{Gadolinium-Based Compounds}

Gadolinium chelates are clinically approved contrast agents that have been used to label cells in experimental cellular MRI studies. When cells are loaded via electroporation, gadolinium locates to the cytoplasm, resulting in a decrease of the T1 relaxation time constant (4). In contrast, incubation of cells with gadolinium results in uptake into endosomes, which shortens the T2 relaxation time constant. Modo et al. (5) tracked the migration of intracerebrally injected stem cells with a combination gadolinium chelate-fluorescent label and were able to detect the presence and migration of cells on T2-weighted images at least $14 \mathrm{~d}$ after injection, with correlative findings by fluorescence microscopy. A transient negative effect of gadolinium-based agents on cell proliferation has been observed; therefore, further evaluation will be needed to ensure that there is no long-term toxicity and that the ability of the cells to repair damage has not been compromised (6). Recently, gadolinium-based agents with substantially greater T1 relaxivities have been developed to label cells. Anderson et al. (7) were able to label mesenchymal stromal cells (MSCs) with gadolinium fullerenol, which has a T1 relaxivity 10 -fold greater than that of gadolinium chelates, and detected an increase in signal intensity on T1-weighted images obtained at $7 \mathrm{~T}$ after direct injection into the rat thigh. However, gadolinium fullerenol labeling initially decreased the stem cell proliferation, suggesting that the agent may be altering mitochondrial function. Thus, there remains a need to identify paramagnetic agents that exert strong T1 effects and allow sufficient detection of cells versus surrounding tissues in circumstances of low numbers of labeled cells or low concentrations of gadolinium.

\section{Fluorinated Nanoparticles}

The main advantage of labeling cells with ${ }^{19} \mathrm{~F}$ perfluorocarbon nanoparticles is the high specificity for labeled cells, because fluorine can be directly detected on MRI and there are no endogenous fluorine atoms in the body. Ahrens et al. (8) labeled dendritic cells (DCs) with cationic perfluoropolyether agents and tracked the migration of the cells to regional lymph nodes (LNs) after injection into the foot pad of mice, using ${ }^{19} \mathrm{~F}$ MRI. Additionally, stem and progenitor cells loaded with perfluorocarbon nanoparticles have been tracked with MRI and MR spectroscopy at both clinical and high field strengths (9). Limitations of this cell-labeling approach include the need for high concentrations of ${ }^{19} \mathrm{~F}$ to achieve a minimal detection threshold, relatively long scanning times, and separate ${ }^{1} \mathrm{H}$ images for anatomic localization of ${ }^{19} \mathrm{~F}$-detected cells.

\section{SPIONS}

SPIONs belong to a family of MRI contrast agents that has seen extensive use to efficiently label cells for imaging. Various methods are used to prepare SPIONs, resulting in a wide range of physiochemical differences including core size, shape, mono- or oligocrystalline composition, and outer coating, all of which alter the biologic activity of SPIONs and the ability to use these agents 
to label cells (2). In addition, micron-sized iron oxide beads ( 0.3 to $>5 \mu \mathrm{m}$ ) incorporating a fluorescent marker are being used to label cells for cellular MRI studies in experimental models (10). Iron perturbs the main magnetic field and can therefore be visualized as signal voids on $\mathrm{T} 2-$ or $\mathrm{T} 2 *$-weighted images. The detection threshold for SPION-labeled cells is affected by magnetic field strength and acquisition parameters. The magnetic susceptibility effects of SPION-labeled cells result in a blooming artifact that extends beyond the size of an individual particle, making the cells conspicuous enough to potentially detect individual cells. The incubation of cells with experimental or clinically approved coated SPION (i.e., ferumoxides, ferucarbotran, or ferumoxtran$10)$, with or without transfection agents, results in iron concentrations from 1 to more than $30 \mathrm{pg}$ of iron per cell, compared with unlabeled cells containing less than approximately $0.1 \mathrm{pg}$ of iron (11). The magnetic labeling of cells with SPIONs has little or no effect on proliferation, metabolic-expression profiles, reactive oxygen species formation, apoptosis rates, or differentiation in the majority of cells studied $(11,12)$. Several techniques have been proposed to increase the sensitivity for iron-labeled cells by directly detecting the magnetic inhomogeneity caused by the iron, using off-resonance or refocusing techniques $(13,14)$; these are termed positive-contrast techniques. However, these same techniques can also be affected by intrinsic susceptibility differences, thereby limiting their utility.

After directly injecting a mixture of ferumoxides and ${ }^{111} \mathrm{In}$ oxine-labeled DCs in the LN of patients with melanoma, de Vries et al. (15) were able to demonstrate the migration of labeled cells by SPECT and MRI through inguinal LNs (Fig. 1). Multimodality imaging allowed for quantification of the number of cells in the LNs, resulting in an MRI detection limit of approximately 2,000 ferumoxide-labeled DCs per voxel based on corresponding measurements by SPECT. Several groups have shown that it is possible to track single magnetically labeled cells by cellular MRI in experimental models $(10,16)$, although it remains to be seen whether these techniques will translate to the clinic.

\section{Magnetic Reporter Genes}

Investigators have recently inserted magnetic reporter genes into cells. The resulting expression of iron storage proteins allows stored iron to be detected by MRI. Genove et al. (17) used an adenoviral vector carrying a transgene for light- and heavy-chain ferritin protein to transfect cells, permitting the detection of cells by MRI in both in vitro and in vivo models. Cohen et al. (18) generated transgenic mice with tissue-specific, inducible transcriptional regulation, using the tetracycline transactivator gene to express heavy-chain ferritin. These authors were able to demonstrate tetracycline-modulated ferritin concentration in the liver and endothelial cells in the brain as measured by $\mathrm{T} 2$ relaxation with MRI. Because the compound is not diluted as the cells divide, the sensitivity to ferritin-expressing cells is not lost. Recently, Zurkiya et al. (19) transfected cells with genes from magnetotactic bacteria (i.e., MagA) under doxycycline-regulated gene expression, resulting in the intracellular production of iron oxide nanoparticles similar to synthetic SPION. MagA-expressing cells could be visualized by MRI after transplantation in the mouse brain after $5 \mathrm{~d}$ of induction with doxycycline. The generalized implementation of these techniques as treatment strategies in stem cell tracking needs to be explored.

\section{APPLICATIONS}

SPIONs have been used extensively to track the migration of stem cells (20-22), immune cells (i.e., DCs, splenocytes, or lymphocytes) (23), and many other cell types $(24,25)$ in numerous animal models of malignancy, angiogenesis, ischemia and infarction, organ failure, autoimmune diseases, and transplantation rejection. Magnetically labeled neural stem cells (NSCs) or neural

\begin{abstract}
FIGURE 1. Monitoring of in vivo migration of SPIONs and $111 \mathrm{In}$ labeled DCs with $\mathrm{MRI}$ and SPECT after intranodal injection. (A, B, E, and H) T2*-weighted gradient-echo (GE) transverse images before vaccination, showing right inguinal $\mathrm{LNs}$ with high signal intensity (arrows). (C, F, and I) T2-weighted spin-echo images after vaccination show $L N s$ with dark-gray signal intensity. (D, G, and J) On GE images after vaccination, all these LNs show decreased signal intensity. (K) Ex vivo scintigraphy of resected LN basin verified MRI findings. From this scintigraph, percentage of $\mathrm{mi}-$ grated cells could be calculated (LN 1, 60\%; LN 2, 32\%; LN 3, 2\%; and LN $4,6 \%$ of the $7.5 \times 10^{6}$ injected DCs). (Adapted with permission of (15).)
\end{abstract}
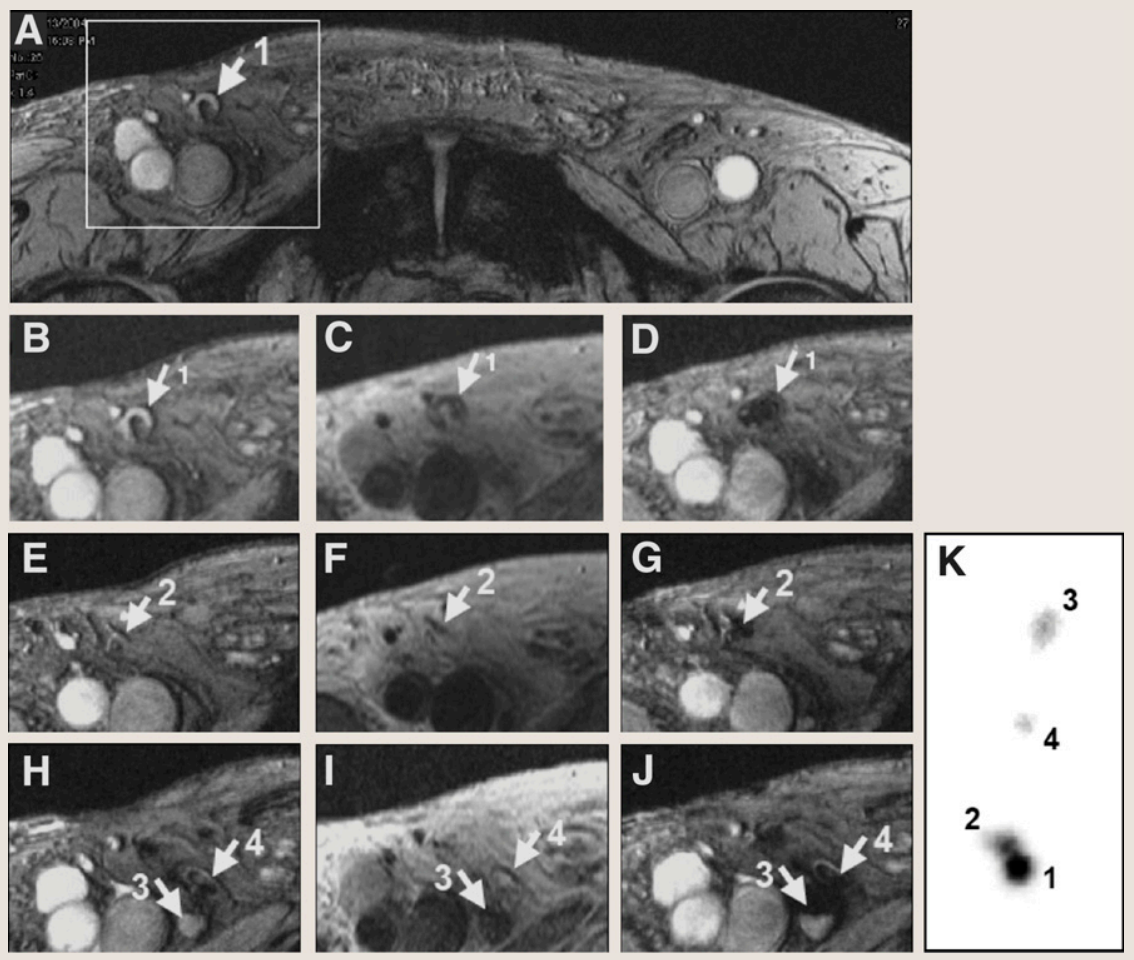
progenitor cells have been implanted in the normal brains of neonatal or adult animals, in stroke $(5,22)$, traumatic brain injury (26), or spinal cord injuries (27). Guzman et al. (21) followed SPION-labeled NSC migration and site-specific differentiation in neonatal and adult rodent brains and in a brain injury model. We have demonstrated the incorporation of SPION-labeled mouse endothelial progenitor cells into the growing neovasculature of tumors in intracerebral (28) and flank tumor models (Fig. 2). Wu et al. (23) demonstrated that the infiltration of labeled cells was a robust marker of cardiac transplant rejection. Kraitchman et al. (20) infused dual-labeled MSCs ( ${ }^{111}$ In-oxine and ferumoxides) and reported focal and diffuse uptake in the infarcted myocardium by SPECT/CT. However, magnetically labeled MSCs detected around the infarction on histology were not observed on MRI. This study demonstrates the need to develop multimodal techniques to detect the presence of distributed labeled cells within target tissues.

The incorporation of magnetically labeled islet cells under the kidney capsule or infused in the portal vein has allowed for the noninvasive monitoring of the treatment of type 1 diabetes in

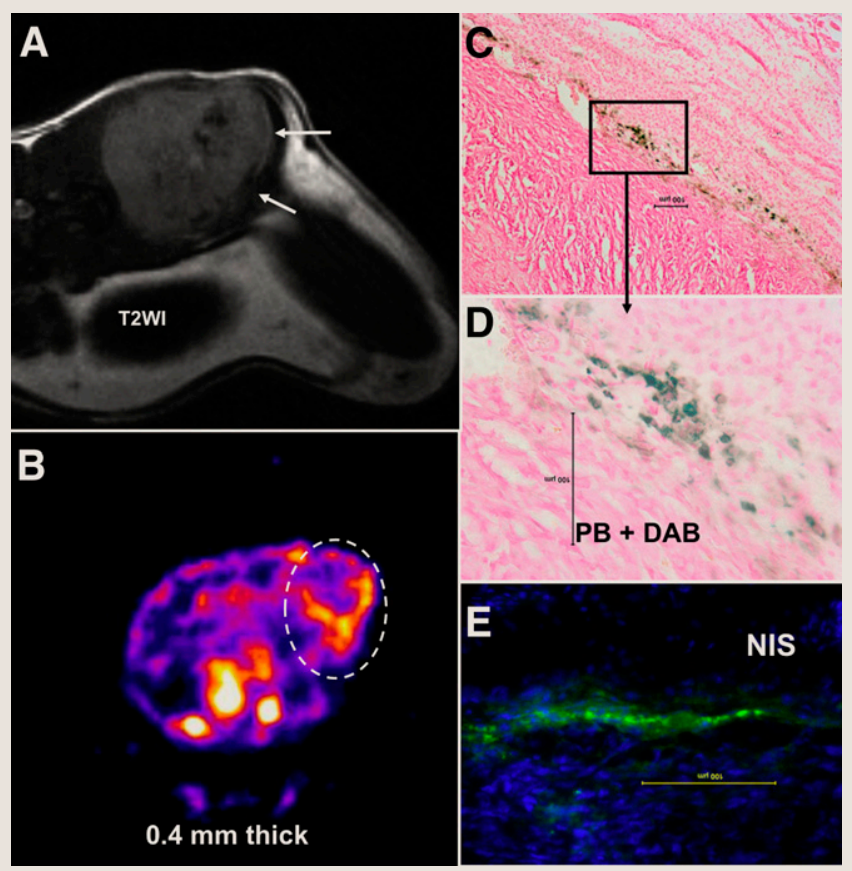

FIGURE 2. Accumulation of magnetically labeled, transgenic endothelial progenitor cells (EPCs) around implanted tumor. T2-weighted MR image shows low-signal-intensity areas at margin of tumor ( $\mathrm{A}$; arrows) that correspond to sites of iron-positive cells detected by Prussian blue staining $(C$ and $D)$. Central hypointense areas are due to hemorrhage within tumor. SPECT image (B; transaxial sections) indicates accumulated transgenic EPCs (white dotted oval region of interest) detected by ${ }^{99 m T}$. SPECT study also confirms migration and homing of EPCs at margin of tumors seen on MRI. Immunohistochemistry depicts accumulation of sodium iodide symporter-positive cells at corresponding sites, detected by anti-hNIS antibody and fluorescein isothiocyanatelabeled secondary antibodies (E). Findings prove that EPCs can carry reporter or therapeutic genes to site of interest and magnetically labeled EPCs act as probe for cellular MRI. PB = Prussian blue. (Images provided courtesy of Arbab Ali, Department of Radiology, Henry Ford Health System.) mouse models (29). Magnetically labeled islets maintained normoglycemia in the animals, and MRI could detect these cells for up to $180 \mathrm{~d}$ after implantation. Serial MRI of islet cell rejection in the liver of mice has been demonstrated by labeling cells with SPIONs (30) and demonstrates the potential value of this technique in monitoring allogenic transplants. These and many other preclinical studies have demonstrated the potential translation of SPION labeling and cellular MRI to the clinic.

There are 4 clinical studies in the literature involving magnetically labeled cells and MRI to monitor the migration of cells to target tissue. The first reported trial involved the injection of magnetically labeled DCs into LNs of patients with melanoma as part of a phase I vaccine study (15). MRI was used to visualize the migration of cells to other LNs within $2 \mathrm{~d}$ (Fig. 1). MRI also demonstrated that the injections of labeled cells missed targeted LNs in 4 patients, indicating the utility and importance of highresolution noninvasive imaging in treatment planning. Zhu et al. (26) cultured and labeled NSCs obtained from patients with open head trauma. Patients received intracerebral injections of either ferumoxide-labeled or unlabeled cells around the injured tissue. Serial MRI up to $7 \mathrm{wk}$ demonstrated the migration of magnetically labeled NSCs from injection sites as hypointense voxels. Neurologic complications were not reported in patients receiving labeled cells. In patients with spinal cord injury, autologous bone marrow CD34-positive cells labeled with magnetic beads could be monitored homing to the injury site for several weeks after infusion into the cerebral spinal fluid (27). MRI was also used to track cadaveric magnetically labeled pancreatic islet cells infused into the portal vein in patients with type 1 diabetes (25), and hypointense regions on T2-weighted MRI scans of the liver were observed for at least $6 \mathrm{wk}$. Despite the limited applications in human patients, these studies nonetheless provide strong evidence that tracking cells with MRI could have important clinical implications.

Cell tracking with MRI suffers from some common limitations observed with all exogenous cell tagging, including sensitivity of the imaging technique, dilution of the exogenous label with cell division, and potential transfer of the magnetic label to tissue macrophages or bystander cells. Although single cells can be detected in vivo in experimental models, the improvement of MRI hardware and detection techniques may improve the sensitivity to reliably detect the presence of labeled cells in tissue. The concentration of the label dilutes as cells divide, limiting the ability to track cells from a few days to several weeks. However, changes in different MRI parameters over time may indicate the engraftment and functional improvement of the target organs or tissues. The transfer of the SPION to activated macrophages or bystander cells in vivo can potentially complicate the interpretation of in vivo MRI results. However, it has been recently reported that the transfer of SPIONs or the genetic label bromodeoxyuridine from MSCs to activated macrophages represented a relatively small amount, compared with total label injected (31).

\section{FUTURE DIRECTIONS}

Although the most promising agents for use in clinical applications are those that have proven to have little or no effects on cell behavior, the functional status of cells is difficult to monitor by in vivo MRI. For cell therapy, the mere presence of labeled cells detected on MRI does not reflect the viability, proliferation, or differential capacity of the cells. Newer "smart" 
MRI agents hold promise in this regard, because the functional status and location of the cell can be probed (32). The advantages afforded by other noninvasive imaging modalities, such as PET, SPECT, ultrasound, and optical imaging, could be used in conjunction with MRI (33). In this regard, multifunctional agents may also serve to provide detailed information on cell function and viability. Tracking labeled cells with MRI has clearly demonstrated utility in evaluating promising cell-based therapies in preclinical models. Although the techniques for labeling cells using magnetic particles continue to be advanced, the translation to the clinic is expected to occur in the near future and will provide researchers with a valuable tool to monitor cell therapies.

\section{Matthew D. Budde Joseph A. Frank National Institutes of Health Clinical Center Bethesda, Maryland}

\section{ACKNOWLEDGMENT}

This work was supported by the Intramural Research Program of the Clinical Center at the National Institutes of Health.

\section{REFERENCES}

1. Arbab AS, Frank JA. Cellular MRI and its role in stem cell therapy. Regen Med. 2008;3:199-215

2. Wilhelm C, Gazeau F. Universal cell labelling with anionic magnetic nanoparticles. Biomaterials. 2008;29:3161-3174.

3. Modo M, Hoehn M, Bulte JW. Cellular MR imaging. Mol Imaging. 2005;4:143-164.

4. Terreno E, Geninatti Crich S, Belfiore S, et al. Effect of the intracellular localization of a Gd-based imaging probe on the relaxation enhancement of water protons. Magn Reson Med. 2006;55:491-497.

5. Modo M, Mellodew K, Cash D, et al. Mapping transplanted stem cell migration after a stroke: a serial, in vivo magnetic resonance imaging study. Neuroimage. 2004;21:311-317.

6. Brekke C, Morgan SC, Lowe AS, et al. The in vitro effects of a bimodal contrast agent on cellular functions and relaxometry. NMR Biomed. 2007;20:77-89.

7. Anderson SA, Lee KK, Frank JA. Gadolinium-fullerenol as a paramagnetic contrast agent for cellular imaging. Invest Radiol. 2006;41:332-338.

8. Ahrens ET, Flores R, Xu H, Morel PA. In vivo imaging platform for tracking immunotherapeutic cells. Nat Biotechnol. 2005;23:983-987.

9. Partlow KC, Chen J, Brant JA, et al. ${ }^{19} \mathrm{~F}$ magnetic resonance imaging for stem/ progenitor cell tracking with multiple unique perfluorocarbon nanobeacons. FASEB J. 2007;21:1647-1654.

10. Shapiro EM, Sharer K, Skrtic S, Koretsky AP. In vivo detection of single cells by MRI. Magn Reson Med. 2006;55:242-249.

11. Arbab AS, Yocum GT, Rad AM, et al. Labeling of cells with ferumoxidesprotamine sulfate complexes does not inhibit function or differentiation capacity of hematopoietic or mesenchymal stem cells. NMR Biomed. 2005;18:553-559.

12. Pawelczyk E, Arbab AS, Pandit S, Hu E, Frank JA. Expression of transferrin receptor and ferritin following ferumoxides-protamine sulfate labeling of cells: implications for cellular magnetic resonance imaging. NMR Biomed. 2006;19: 581-592.
13. Cunningham CH, Arai T, Yang PC, McConnell MV, Pauly JM, Conolly SM. Positive contrast magnetic resonance imaging of cells labeled with magnetic nanoparticles. Magn Reson Med. 2005;53:999-1005.

14. Liu W, Dahnke H, Jordan EK, Schaeffter T, Frank JA. In vivo MRI using positive-contrast techniques in detection of cells labeled with superparamagnetic iron oxide nanoparticles. NMR Biomed. 2008;21:242-250.

15. De Vries IJ, Lesterhuis WJ, Barentsz JO, et al. Magnetic resonance tracking of dendritic cells in melanoma patients for monitoring of cellular therapy. Nat Biotechnol. 2005;23:1407-1413.

16. Heyn C, Ronald JA, Ramadan SS, et al. In vivo MRI of cancer cell fate at the single-cell level in a mouse model of breast cancer metastasis to the brain. Magn Reson Med. 2006;56:1001-1010.

17. Genove G, DeMarco U, Xu H, Goins WF, Ahrens ET. A new transgene reporter for in vivo magnetic resonance imaging. Nat Med. 2005;11:450-454.

18. Cohen B, Ziv K, Plaks V, et al. MRI detection of transcriptional regulation of gene expression in transgenic mice. Nat Med. 2007;13:498-503.

19. Zurkiya O, Chan AW, Hu X. MagA is sufficient for producing magnetic nanoparticles in mammalian cells, making it an MRI reporter. Magn Reson Med. 2008;59:1225-1231.

20. Kraitchman DL, Tatsumi M, Gilson WD, et al. Dynamic imaging of allogeneic mesenchymal stem cells trafficking to myocardial infarction. Circulation. 2005;112:1451-1461.

21. Guzman R, Uchida N, Bliss TM, et al. Long-term monitoring of transplanted human neural stem cells in developmental and pathological contexts with MRI. Proc Natl Acad Sci USA. 2007;104:10211-10216.

22. Walczak P, Zhang J, Gilad AA, et al. Dual-modality monitoring of targeted intraarterial delivery of mesenchymal stem cells after transient ischemia. Stroke. 2008;39:1569-1574.

23. Wu YL, Ye Q, Foley LM, et al. In situ labeling of immune cells with iron oxide particles: an approach to detect organ rejection by cellular MRI. Proc Natl Acad Sci USA. 2006;103:1852-1857.

24. Foster PJ, Dunn EA, Karl KE, et al. Cellular magnetic resonance imaging: in vivo imaging of melanoma cells in lymph nodes of mice. Neoplasia. 2008;10: 207-216.

25. Toso C, Vallee JP, Morel $\mathrm{P}$, et al. Clinical magnetic resonance imaging of pancreatic islet grafts after iron nanoparticle labeling. Am J Transplant. 2008;8: 701-706.

26. Zhu J, Zhou L, XingWu F. Tracking neural stem cells in patients with brain trauma. N Engl J Med. 2006;355:2376-2378.

27. Callera F, de Melo CM. Magnetic resonance tracking of magnetically labeled autologous bone marrow CD34+ cells transplanted into the spinal cord via lumbar puncture technique in patients with chronic spinal cord injury: CD34+ cells' migration into the injured site. Stem Cells Dev. 2007;16:461-466.

28. Anderson SA, Glod J, Arbab AS, et al. Noninvasive MR imaging of magnetically labeled stem cells to directly identify neovasculature in a glioma model. Blood. 2005; 105:420-425.

29. Evgenov NV, Medarova Z, Dai G, Bonner-Weir S, Moore A. In vivo imaging of islet transplantation. Nat Med. 2006;12:144-148.

30. Evgenov NV, Medarova Z, Pratt J, et al. In vivo imaging of immune rejection in transplanted pancreatic islets. Diabetes. 2006;55:2419-2428.

31. Pawelczyk E, Arbab AS, Chaudhry A, Balakumaran A, Robey PG, Frank JA. In vitro model of bromodeoxyuridine or iron oxide nanoparticle uptake by activated macrophages from labeled stem cells: implications for cellular therapy. Stem Cells. 2008;26:1366-1375.

32. Gilad AA, McMahon MT, Walczak P, et al. Artificial reporter gene providing MRI contrast based on proton exchange. Nat Biotechnol. 2007;25:217-219.

33. Ly HQ, Frangioni JV, Hajjar RJ. Imaging in cardiac cell-based therapy: in vivo tracking of the biological fate of therapeutic cells. Nat Clin Pract Cardiovasc Med. 2008;5(suppl 2):S96-S102. 